\title{
Milnor number at infinity, topology and Newton boundary of a polynomial function
}

\author{
E. Artal Bartolo ${ }^{1}$, I. Luengo ${ }^{2}$, A. Melle-Hernández ${ }^{2}$ \\ 1 Departamento de Matemáticas, Universidad de Zaragoza, Campus Plaza San Francisco \\ s/n, E-50009, Zaragoza, Spain (e-mail: artal@ posta.unizar.es) \\ 2 Departamento de Álgebra, Universidad Complutense, Ciudad Universitaria s/n, E-28040, \\ Madrid, Spain (e-mail: iluengo@eucmos.sim.ucm.es, amelle@eucmos.sim.ucm.es)
}

\begin{abstract}
In this paper we show that the Euler characteristic of the generic fibre of a complex polynomial function $f: \mathbb{C}^{n} \rightarrow \mathbb{C}$ can be easily computed using the Newton number of $f$. We apply this result to study polynomials with a finite number of critical points.
\end{abstract}

Mathematics Subject Classification (1991): 14F45, 14D25, 32S15, 32S60.

Let $f: \mathbb{C}^{n} \rightarrow \mathbb{C}$ be a complex polynomial function and let $B_{f} \subset \mathbb{C}$ be its bifurcation set, i.e. the (smallest) finite set of atypical values $t \in \mathbb{C}$ where $f$ is not a $C^{\infty}$-locally trivial fibration, ([HL], [V], [Ve]). The bifurcation set $B_{f}$ includes not only the set of critical values $\Sigma_{f}$ of $f$ but $B_{f}$ may also contain values corresponding to the critical points at infinity; these are called atypical values at infinity, for a definition see e.g. [GLM1].

In a neighborhood of an atypical value at infinity the topology of the fibres $F_{t}:=f^{-1}(t)$ of the function $f$ changes. This change is well understood when the singularities of the compactifications $\bar{F}_{t} \subset \mathbb{P}^{n}$ of the fibres are isolated (cf. [C], [Du],[HL],[P3]).

In the paper we mostly deal with complex polynomials with only isolated critical points and we allow the singularities at infinity to be non-isolated. In such a case let $\mu(f)$ be the sum of the local Milnor numbers of the germs of the level hypersurfaces of $f$ at its singular points and let $\mu_{f}^{a}(t)$ be the sum of the local Milnor numbers of the germs of the fibre $F_{t}$ at its singular

This work has been done when the authors had the Proyecto de Investigación Español with reference number CAICYT PB94-0291 and the projects DGES PB97-0284-C02-02 (for the first author) and DGES PB97-0284-C02-01 (for the other ones). 
points. We will define later invariants $\lambda(f)$ and $\lambda_{f}(b), b \in \mathbb{C}$, as a difference between generalized Milnor numbers at infinity of the special fibres and of the generic one. One computes explicitly the Euler characteristic of the fibres $F_{t}, t \in \mathbb{C}$, as follows.

Theorem. Let $f: \mathbb{C}^{n} \rightarrow \mathbb{C}$ be a polynomial function having only isolated critical points.

(i) Ift $\in \mathbb{C} \backslash B_{f}$ then the Euler characteristic $\chi\left(F_{t}\right)$ of the fibre $F_{t}$ can be computed as

$$
\chi\left(F_{t}\right)=1+(-1)^{n-1}(\mu(f)+\lambda(f)) .
$$

(ii) For any value $b \in \mathbb{C}$ the Euler characteristic $\chi\left(F_{b}\right)$ of the fibre $F_{b}$ satisfies

$$
\chi\left(F_{b}\right)=\chi\left(F_{t}\right)+(-1)^{n}\left(\mu_{f}^{a}(b)+\lambda_{f}(b)\right) .
$$

As a consequence of this theorem the invariant $\lambda(f)$ coincides with the invariant defined by H.V. Hà and D.T. Lê, [HL], for polynomials in two variables and Siersma and Tibăr, [ST], for polynomials with $\mathcal{W}$-isolated singularities at infinity. Recently, Tibăr [T] has used the equalities $(i)$ and (ii) as definitions of the invariants $\lambda(f)$ and $\lambda_{f}(b)$. Our approach is slightly different. We define the invariants $\lambda(f)$ and $\lambda_{f}(b)$ using the generalized Milnor number introduced by Parusiński in [P1]. The main reason to do this is that $\lambda(f)$ and $\lambda_{f}(b)$ can be explicitly computed in many cases using the properties of the generalized Milnor number.

In case $f$ has non-degenerated Newton principal part at infinity, (see $\S 2$ for definitions), the Euler characteristic of the generic fibre of $f$ is computed in terms of the Newton number of $f$. In particular, if the polynomial function $f: \mathbb{C}^{n} \rightarrow \mathbb{C}$ has only isolated critical points then the invariant $\lambda(f)$ is related to its Newton number $\nu(f)$. This fact gives the main result of the paper:

Theorem. Let $f: \mathbb{C}^{n} \rightarrow \mathbb{C}$ be a complex polynomial function with only isolated critical points and non-degenerated Newton principal part at infinity, then

$$
\mu(f)+\lambda(f)=\nu(f) .
$$

This result is a generalization of Kushnirenko's Theorem, [Ku], namely, if $f$ is also convenient then $\lambda(f)=0$ and the above theorem gives the equality in Kushnirenko's Theorem. In fact, the apparently striking relationship between $\mu(f)$ and $\nu(f)$ can be explained from the fact that $\lambda(f)$ may be negative when $n>2$ and the singularities of the fibres are not isolated at infinity. Cassou-Noguès in $[\mathrm{C}]$ proved the theorem for a complex polynomial function in two variables; she also got the inequality $\leq$ for polynomials with degenerated Newton principal part at infinity. As a corollary of our results 
we prove her Conjecture, [C, p. 120], in the non-degenerated case not only for tame polynomials, [B], but for any polynomial having $\lambda(f)=0$, e.g. quasi-tame or M-tame polynomials, [N1], [NZ]. Related with this conjecture there are some new results about the positivity of $\lambda(f)+\lambda\left(f_{\mid H}\right)$ (for $H$ a generic hyperplane) in [CD].

Finally, we give a list of interesting examples. Example 3.1 disproves Cassou-Noguès' conjecture in the degenerated case. In Example 3.8 we study the fibres of $x+x^{2} y z+u^{k}+v^{l}$. We conjecture that its zero fibre is an exotic $\mathbb{C}^{4}$. A negative answer to this conjecture would give a counterexample to the Abhyankar-Shataye conjecture in dimensions 4 and 5 .

Other interesting invariants of a complex polynomial function $f$ besides the integers $\lambda_{f}(t)$ and $\lambda(f)$ are the zeta-functions of the local monodromies around the atypical values (including the $\infty$ itself). These zeta-functions (and therefore the Euler characteristic of the fibres, $\lambda_{f}(t)$ and $\lambda(f)$ ) can be also computed using germs of meromorphic functions at the points of the hyperplane at infinity, see [GLM1]. Further generalizations of the previous invariants for a meromorphic function on a compact complex manifold have been described in [GLM2].

\section{Polynomials with isolated critical points}

\subsection{Generalized Milnor number}

Let us recall a number of known results about the generalized Milnor number introduced by Parusiński. We denote by $\chi(A)$ the Euler characteristic of $A$.

Let $\mathcal{E}$ be a holomorphic vector bundle of rank $r$ over a smooth compact complex manifold $M$ of dimension $n$. Let $s \in \mathbb{P}\left(H^{0}(M ; \mathcal{E})\right)$ and let $X$ be the zero set of a representative of $s$. Parusiński [P2] defined the Milnor number of $X$ in $M$, denoted by $\mu(M ; X)$, as follows

$$
\mu(M ; X):=(-1)^{n-r+1}(\chi(X)-\chi(M ; \mathcal{E})),
$$

where $\chi(M ; \mathcal{E})$ is the Euler characteristic of the zero set of a section of $\mathcal{E}$ transversal to the zero section.

Let us enumerate some properties of this invariant from [P1] and [PP]:

Property 1.1. [P1] If $X$ and $Z$ are two hypersurfaces equivalent as divisors then

$$
\mu(M ; X)-\mu(M ; Z)=(-1)^{n}(\chi(X)-\chi(Z)) .
$$

Property 1.2. [PP] The generalized Milnor number can be computed as follows. Let $X$ be a hypersurface on $M$. If $f_{x}=0$ is a local equation of $X$ at $x \in X$ the topological Milnor number of $X$ at $x$ is

$$
\mu_{\text {top }}(X, x):=(-1)^{n-1}\left(\chi\left(F_{x}\right)-1\right),
$$


where $F_{x}$ is the Milnor fibre of $f_{x}$ at the point $x$; it is just the classical Milnor number if $x$ is an isolated singular point of $X$.

Given a Whitney stratification $\mathcal{S}$ of $X$ the topological Milnor number $\mu_{\text {top }}(X, x)$ has a constant value along each stratum; let us denote by $\mu_{S}$ this value for each stratum $S \in \mathcal{S}$. If $Z$ is a smooth hypersurface on $M$ linearly equivalent to $X$ and transversal to $\mathcal{S}$ then the Milnor number of $X$ in $M$ verifies the equality

$$
\mu(M ; X)=\sum_{S \in \mathcal{S}} \chi(S \backslash Z) \mu_{S} .
$$

Remark. In [P1] it is defined a more general invariant $\mu(M ; X, Y)$, where $Y$ is a compact subvariety of $X$ which admits a neighborhood $U$ in $X$ such that $U \backslash Y$ is nonsingular. If $Z$ and $\mathcal{S}$ are as above and $\mathcal{S}$ induces a stratification $\mathcal{S}_{Y}$ on $Y$ then

$$
\mu(M ; X, Y)=\sum_{S \in \mathcal{S}_{Y}} \chi(S \backslash Z) \mu_{S}
$$

Let $\mathcal{L}$ be a holomorphic line bundle over a smooth compact complex manifold $M$ and let $V$ be a $k$-dimensional subspace of the projective space of the global sections $\mathbb{P}\left(H^{0}(M ; \mathcal{L})\right)$. For any $v \in V$, let $X_{v}$ be the zero set of a representative of $v$. There exists a stratification $\mathcal{S}$ of $V$ such that $\mu\left(M ; X_{v}\right)$ is constant along each stratum of $\mathcal{S}$; for any $S \in \mathcal{S}$ we denote by $\mu_{S}$ this constant Milnor number.

Proposition 1.3. [P2] Let $V$ and $\mathcal{S}$ be as above and let $B$ be the base points of $V$ (considered as the zero set of a section of $\mathcal{L}^{\oplus k+1}$ ). Then the integer

$$
\gamma_{k}(\mathcal{L}):=\sum_{S \in \mathcal{S}} \chi(S) \mu_{S}+(-1)^{k+1} \mu(M ; B)
$$

only depends on $k$ and $\mathcal{L}$.

\subsection{Milnor number at infinity and the $\lambda$-invariant}

Let $f: \mathbb{C}^{n} \rightarrow \mathbb{C}$ be a complex polynomial function with only isolated critical points and let $f=f_{0}+f_{1}+\cdots+f_{d}$ be its decomposition in homogeneous forms. We take the natural inclusion $\mathbb{C}^{n} \hookrightarrow \mathbb{P}^{n}$; we will denote by $\mathbb{P}_{\infty}^{n-1}$ the hyperplane at infinity defined by $x_{0}=0$. Let $D$ be the divisor of $\mathbb{P}_{\infty}^{n-1}$ defined by the zero locus of the homogeneous polynomial $f_{d}$. We will denote by $\bar{F}_{t}$ the compactification of $F_{t}:=f^{-1}(t)$ in $\mathbb{P}^{n}$; the equation of $\bar{F}_{t}$ is $\tilde{f}\left(x_{0}, x_{1}, \ldots, x_{n}\right)-t x_{0}^{d}=0$, where $\tilde{f}:=f_{d}+x_{0} f_{d-1}+\cdots+x_{0}^{d-1} f_{1}+x_{0}^{d} f_{0}$. 
Since the set $\mathrm{C}(f)$ of critical points is finite then the sum of the local Milnor numbers of the germs of the level hypersurfaces of $f$ at the points of $\mathrm{C}(f)$ is a finite integer denoted by $\mu(f)$.

Let us consider the function $\mu_{f}^{\infty} \mathbb{C} \rightarrow \mathbb{Z}$, where $t \mapsto \mu\left(\mathbb{P}^{n} ; \bar{F}_{t}, D\right)$. If it is constant we will denote its constant value by $\mu_{f}^{\infty}$.

One can apply the invariant $\gamma_{k}(\mathcal{L})$ of $(1.3)$ to this case. Such a polynomial is interpreted as a 1-dimensional subspace $\mathbb{P}_{f}^{1}$ of $H^{0}\left(\mathbb{P}^{n}, \mathcal{O}(d)\right)$ generated by the $d^{\text {th }}$-homogeneous polynomials $\tilde{f}$ and $x_{0}^{d}$. The zero sets associated to $\mathbb{P}_{f}^{1}=\mathbb{C} \cup\{\infty\}$ are the hypersurfaces $\bar{F}_{t}, t \in \mathbb{C}$, and $d$ times $\mathbb{P}_{\infty}^{n-1}$.

The fact that the function $f$ has only isolated critical points allows to separate the affine singular points of each $\bar{F}_{t}$ from the singularities at infinity. Namely, for each $t \in \mathbb{C}$ the generalized Milnor number $\mu\left(\mathbb{P}^{n} ; \bar{F}_{t}\right)$ of $\bar{F}_{t}$ in $\mathbb{P}^{n}$ can be expressed as

$$
\mu\left(\mathbb{P}^{n} ; \bar{F}_{t}\right)=\mu\left(\mathbb{P}^{n} ; \bar{F}_{t}, D\right)+\mu\left(\mathbb{P}^{n} ; \bar{F}_{t}, \operatorname{Sing}\left(F_{t}\right)\right) .
$$

Let us denote $\mu\left(\mathbb{P}^{n} ; \bar{F}_{t}, \operatorname{Sing}\left(F_{t}\right)\right)$ by $\mu_{f}^{a}(t)$; recall that $\mu\left(\mathbb{P}^{n} ; \bar{F}_{t}, D\right)=$ $\mu_{f}^{\infty}(t)$.

Two useful stratifications. In order to stratify $\mathbb{P}_{f}^{1}$ in a way such that the generalized Milnor number of $\bar{F}_{t}$ is constant over each stratum we consider the following stratifications of $\mathbb{C}=\mathbb{P}_{f}^{1} \backslash\{\infty\}$ :

(1) We choose a stratification $\mathcal{S}_{a}$ of $\mathbb{C}$ such that $\mu_{f}^{a}(t)$ is constant of value $\mu_{S}^{a}$ along the stratum $S$ of $\mathcal{S}_{a}$. Because of Bertini theorem $\mu_{S_{0}}^{a}$ vanishes if $S_{0}$ is the 1-dimensional stratum and

$$
\sum_{S \in \mathcal{S}_{a}} \mu_{S}^{a} \chi(S)=\mu(f)
$$

(2) Since $\mathrm{C}(f)$ is finite one takes any stratification $\mathcal{S}_{\infty}$ of $\mathbb{C}$ such that $\mu_{f}^{\infty}(t)$ is constant along each stratum $S$ of $\mathcal{S}_{\infty}$. Let $\mu_{S}^{\infty}$ be this constant value over the stratum $S \in \mathcal{S}_{\infty}$. Let $S_{\text {gen }}:=\mathbb{C} \backslash\left\{t_{1}, \ldots, t_{m}\right\}$ be the 1dimensional stratum of $\mathcal{S}_{\infty}$ and let $\mu_{g e n}^{\infty}(f)$ be the value of $\mu_{f}^{\infty}$ on $S_{g e n}$.

Definition 1.4. With the above notations we define for any $t \in \mathbb{C}$

$$
\lambda_{f}(t):=\mu_{f}^{\infty}(t)-\mu_{g e n}^{\infty}(f)
$$

and

$$
\lambda(f):=\sum_{t \in \mathbb{C}} \lambda_{f}(t)=\sum_{j=1}^{m} \lambda_{f}\left(t_{j}\right) .
$$


It is easily seen that $\lambda_{f}(t)$ and $\lambda(f)$ do not depend on the choice of the stratification verifying $(2)$. Moreover

$$
\sum_{S \in \mathcal{S}_{\infty}} \chi(S) \mu_{S}^{\infty}=\lambda(f)+\mu_{g e n}^{\infty}(f)
$$

and it is clear that if the function $\mu_{f}^{\infty}$ is constant then $\lambda(f)$ is equal to zero.

Let $S$ be the non-empty open set $S:=S_{0} \cap S_{\text {gen }}=\mathbb{C} \backslash\left\{s_{1}, \ldots, s_{k}\right\}$. Let $\mathcal{S}$ be the following stratification of $\mathbb{P}_{f}^{1}: S,\left\{s_{i}\right\}_{i=1}^{k}$ and $\{\infty\}$. It is easy to see that the stratification $\mathcal{S}$ verifies the condition of (2) and for all $t \in S$ the integer $\mu\left(\mathbb{P}^{n} ; \bar{F}_{t}\right)$ is constant.

Example 1.5. Let us consider $f(x, y):=x+x^{2} y \in \mathbb{C}[x, y]$. Choosing coordinates $[X: Y: Z]$ in $\mathbb{P}^{2}(x=X / Z, y=Y / Z)$ one has $D_{\text {red }}:=$ $\left\{P_{1}=[1: 0: 0], P_{2}=[0: 1: 0]\right\} \subset \mathbb{P}_{\infty}^{1}$. We compute the (generalized) Milnor number of $\bar{F}_{t}$ at infinity. It is obvious that $\mu_{f}^{\infty}(t)=\mu\left(\mathbb{P}^{2} ; \bar{F}_{t}, P_{1}\right)+$ $\mu\left(\mathbb{P}^{2} ; \bar{F}_{t}, P_{2}\right)$. Let $Z=0$ be the equation of the line at infinity. Then the local equation of $\bar{F}_{t}$ at $P_{1}$ is $y+z^{2}-t z^{3}=0$; it is smooth and $\mu\left(\mathbb{P}^{2} ; \bar{F}_{t}, P_{1}\right)=0$ for all $t \in \mathbb{C}$. Analogously, the local equation of $\bar{F}_{t}$ at $P_{2}$ is $x^{2}+x z^{2}-t z^{3}=0$; then $\mu\left(\mathbb{P}^{2} ; \bar{F}_{t}, P_{2}\right)=2$ for all $t \in \mathbb{C}^{*}$ and $\mu\left(\mathbb{P}^{2} ; \bar{F}_{0}, P_{2}\right)=3$. Taking the stratification $\mathcal{S}_{\infty}:=\left\{\{0\}, \mathbb{C}^{*}\right\}$ one gets $\lambda(f)=1$.

Next consider $f(x, y)$ as a polynomial function $f: \mathbb{C}^{3} \rightarrow \mathbb{C}$. Then $D$ is the projective curve in $\mathbb{P}_{\infty}^{2}=\{w=0\}$ whose equation is $x^{2} y=0$. In order to compute $\mu\left(\mathbb{P}^{3} ; \bar{F}_{t}, D\right)$ we apply the remark after Property (1.2). We choose as stratification $\mathcal{S}_{D}$ the canonical Whitney stratification of $D_{\text {red }}$. We will see that it matches with the required properties.

Let $S_{y}$ be the stratum of equation $y=w=0, x \neq 0$. Since $\bar{F}_{t}$ is smooth at any point $[1: 0: z: 0]$ in this stratum then

$$
\mu_{\text {top }}\left(\bar{F}_{t},[1: 0: z: 0]\right)=\mu\left(\mathbb{P}^{3} ; \bar{F}_{t},[1: 0: z: 0]\right)=0
$$

for all $t \in \mathbb{C}$ which yields $\mu_{S_{y}}=0$.

Let $S_{x}$ be the stratum of equation $x=w=0, y \neq 0$. The Milnor fibre of $\bar{F}_{t}$ at any point $[0: 1: z: 0]$ is the product of the Milnor fibre in two variables by a disk, so the Euler characteristic does not change along the stratum and $\mu_{\text {top }}\left(\bar{F}_{t},[0: 1: z: 0]\right)=-2$ for all $t \in \mathbb{C}^{*}, \mu_{\text {top }}\left(\bar{F}_{0},[0: 1: z: 0]\right)=-3$. Then $\mu_{S_{x}}$ is well defined.

Finally, let us consider the one-point stratum $S=\{[0: 0: 1: 0]\}$. We compute the Milnor number of the homogeneous non-isolated singularity $x w^{2}+x^{2} y-t w^{3}=0$ at $(0,0,0)$. Its Milnor fibre is the 3 -fold cyclic covering of the complement in $\mathbb{P}^{2}$ of the corresponding projective curve. It means that the Euler characteristic of the Milnor fibre of $\bar{F}_{t}$ is equal to 0 if $t=0$ and 3 otherwise. Then $\mu_{\text {top }}\left(\bar{F}_{t},[0: 0: 1: 0]\right)=2$ for all $t \in \mathbb{C}^{*}$ and $\mu_{\text {top }}\left(\bar{F}_{0},[0: 0: 1: 0]\right)=-1$. 
In order to compute the formula we choose a generic cubic surface $Z$ in $\mathbb{P}^{3}$. It is easily seen that $S \backslash Z=S$, and $S_{x}$ and $S_{y}$ are homeomorphic to $\mathbb{C}$ minus three points. Then the formula gives

$$
\mu\left(\mathbb{P}^{3} ; \bar{F}_{t}, D\right)=\mu_{S} \chi(S \backslash Z)+\mu_{S_{x}} \chi\left(S_{x} \backslash Z\right)+\mu_{S_{y}} \chi\left(S_{y} \backslash Z\right)
$$

gives $\mu\left(\mathbb{P}^{3} ; \bar{F}_{t}, D\right)=6$ for all $t \in \mathbb{C}^{*}$ and $\mu\left(\mathbb{P}^{2} ; \bar{F}_{0}, D\right)=5$. It follows that $\lambda(f)=-1$.

More general computations of $\lambda$ can be found in [ALM].

Corollary 1.6. Let $f: \mathbb{C}^{n} \rightarrow \mathbb{C}$ be a polynomial function with a finite number of critical points. Let d be the degree of the polynomial $f$. If $\mathcal{S}_{\infty}$ is as above then the integer

$$
\gamma(d, n):=\mu(f)+\lambda(f)+\mu_{g e n}^{\infty}(f)+(-1)^{n-1} \chi(D)
$$

only depends on $d$ and $n$.

Proof. From (1.3) the integer

$$
\gamma_{1}(\mathcal{O}(d))=\mu(f)+\sum_{\mathcal{S}} \chi(S) \mu_{S}^{\infty}+\mu\left(\mathbb{P}^{n} ; d \mathbb{P}^{n-1}\right)+\mu\left(\mathbb{P}^{n} ; D\right)
$$

only depends on $\mathcal{O}(d)$ where $D$ is considered as the zero set of a section of $\mathcal{O}(d) \oplus \mathcal{O}(d)$. We recall that

$$
\mu\left(\mathbb{P}^{n} ; D\right)=(-1)^{n-1}\left(\chi(D)-\chi\left(\mathbb{P}^{n} ; \mathcal{O}(d) \oplus \mathcal{O}(d)\right)\right) .
$$

Since $\mu\left(\mathbb{P}^{n} ; d \mathbb{P}^{n-1}\right)$ and $\chi\left(\mathbb{P}^{n} ; \mathcal{O}(d) \oplus \mathcal{O}(d)\right)$ only depend on $\mathcal{O}(d)$ the corollary is proved.

Theorem 1.7. Let $f: \mathbb{C}^{n} \rightarrow \mathbb{C}$ be a polynomial function with a finite number of critical points. Let $t \in \mathbb{C} \backslash B_{f}$ then

(i) The Euler characteristic $\chi\left(F_{t}\right)$ of the fiber $F_{t}$ satisfies

$$
\chi\left(F_{t}\right)=1+(-1)^{n-1}(\mu(f)+\lambda(f)) .
$$

(ii) For any value $b \in \mathbb{C}$ the Euler characteristic $\chi\left(F_{b}\right)$ of the fibre $F_{b}$ is equal to

$$
\chi\left(F_{b}\right)=\chi\left(F_{t}\right)+(-1)^{n}\left(\mu_{f}^{a}(b)+\lambda_{f}(b)\right) .
$$


Remark. This theorem is a generalization of Corollary 3.5 of [ST] which applies only for polynomials with $\mathcal{W}$-isolated singularities at infinity. Therefore the above definition of $\lambda(f)$ is a natural generalization of other invariants defined in [C] and [ST]. We note also that $\lambda(f)$ is invariant up to polynomial automorphism. As the above example has shown $\lambda(f)$ could be a negative integer.

Proof. (i) Let us take the polynomial function $g:=x_{1}^{d}+\cdots+x_{n}^{d}: \mathbb{C}^{n} \rightarrow$ $\mathbb{C}$. The origin is its only critical point and $\mu(g)=(d-1)^{n}$. Moreover $\mu_{g}^{\infty}(t)=0 \forall t \in \mathbb{C}$. The intersection of the compactified fibres of $g$ with the hyperplane at infinity is a smooth hypersurface $D_{g} \subset \mathbb{P}_{\infty}^{n-1}$ of degree $d$. Since $\mu_{g}^{\infty}$ has constant value equal to zero one takes the trivial stratification of $\mathbb{C}$ for $g$. Moreover (1.6) shows that

$$
\mu(f)+\lambda(f)+\mu_{g e n}^{\infty}(f)+(-1)^{n-1} \chi(D)=(d-1)^{n}+(-1)^{n-1} \chi\left(D_{g}\right) .
$$

Let $V_{g}$ be the compactification of $g^{-1}(0)$ and let $t \in \mathbb{C} \backslash B_{f}$ be a generic value then $\mu\left(\mathbb{P}^{n} ; \bar{F}_{t}\right)=\mu_{g e n}^{\infty}(f), \mu\left(\mathbb{P}^{n}, V_{g}\right)=(d-1)^{n}$ and $\chi\left(V_{g}\right)=$ $1+\chi\left(D_{g}\right)$. The above formula becomes

$$
\begin{aligned}
& \mu(f)+\lambda(f)+\mu\left(\mathbb{P}^{n} ; \bar{F}_{t}\right)+(-1)^{n-1} \chi(D) \\
& =\mu\left(\mathbb{P}^{n} ; V_{g}\right)+(-1)^{n-1}\left(\chi\left(V_{g}\right)-1\right) .
\end{aligned}
$$

Using the properties of the generalized Milnor number one gets

$$
\mu\left(\mathbb{P}^{n} ; V_{g}\right)-\mu\left(\mathbb{P}^{n} ; \bar{F}_{t}\right)=(-1)^{n}\left(\chi\left(V_{g}\right)-\chi\left(\bar{F}_{t}\right)\right)
$$

and then

$$
\mu(f)+\lambda(f)+(-1)^{n-1} \chi(D)=(-1)^{n-1}\left(\chi\left(\bar{F}_{t}\right)-1\right) .
$$

It is clear that $\chi\left(\bar{F}_{t}\right)=\chi\left(F_{t}\right)+\chi(D)$ and the result of the first part follows.

(ii) Let $b \in \mathbb{C}$. It is clear that $\chi\left(\bar{F}_{b}\right)-\chi\left(\bar{F}_{t}\right)=\chi\left(F_{b}\right)-\chi\left(F_{t}\right)$. Using first (1.1) and secondly the definition of the generalized Milnor number the following equalities follow

$$
\begin{aligned}
\chi\left(F_{b}\right)-\chi\left(F_{t}\right)= & (-1)^{n}\left(\mu\left(\mathbb{P}^{n} ; \bar{F}_{b}\right)-\mu\left(\mathbb{P}^{n} ; \bar{F}_{t}\right)\right) \\
= & (-1)^{n}\left(\mu\left(\mathbb{P}^{n} ; \bar{F}_{b}, D\right)+\mu\left(\mathbb{P}^{n} ; \bar{F}_{b}, \operatorname{Sing}\left(F_{b}\right)\right)\right. \\
& \left.-\mu\left(\mathbb{P}^{n} ; \bar{F}_{t}, D\right)\right) .
\end{aligned}
$$

So the theorem is proved.

Remark. The negative sign of $\lambda(f)$ in the second example of (1.5) is an easy consequence of this theorem as in such a case $\mu(f)=0$ in 2 and 3 variables and the Euler characteristic of the generic fibre does not change. 
The following results are an easy consequence of the theorem but, indeed, they are very useful for computations.

Corollary 1.8. Let $f: \mathbb{C}^{n} \rightarrow \mathbb{C}$ be a complex polynomial function with a finite number of critical points such that $\mu_{f}^{\infty}$ is constant. If $t \in \mathbb{C} \backslash B(f)$ then

$$
\chi\left(F_{t}\right)=1+(-1)^{n-1} \mu(f) .
$$

Sebastiani-Thom formula for $\lambda(f)$. It is clear from a theorem of Némethi, [N2], and our previous result that there exists a formula of Sebastiani-Thomtype for the invariant $\lambda(f)$. Suppose that $f(\bar{x}, \bar{y})=g(\bar{x})+h(\bar{y}), f: \mathbb{C}^{n} \times$ $\mathbb{C}^{m} \rightarrow \mathbb{C}$. Némethi showed that the generic fibre of $f$ is homotopically equivalent to the join space of the generic fibres of the polynomials $g$ and $h$ (for the definition of join space see e.g. [D]). So if $g$ and $h$ have only isolated critical points then $f$ has only isolated critical points and

(1) $\mu(f)=\mu(g) \mu(h)$ and

(2) $\lambda(f)=\lambda(g) \lambda(h)+\lambda(g) \mu(h)+\lambda(h) \mu(g)$.

Now suppose that $f(x, y)=g(x)$ where $f: \mathbb{C}^{n} \times \mathbb{C}^{m} \rightarrow \mathbb{C}$ and $g: \mathbb{C}^{n} \rightarrow$ $\mathbb{C}$. The polynomial function $f$ has only isolated critical points if and only if $g$ has $\mu(g)=0$. In such a case $\mu(f)=\mu(g)=0$ and $\lambda(f)=(-1)^{m} \lambda(g)$.

For a germ $(V, 0) \subset\left(\mathbb{C}^{n}, 0\right)$ of isolated hypersurface singularity there exists a Noether-type formula relating the following invariants of the singularity $(V, 0)$ : its local Milnor number, its multiplicity, the generalized Milnor number of its tangent cone and the generalized Milnor number of its strict transform after one blowing-up, see e.g. [Me]. Next theorem shows a similar Noether-type formula in the global case.

Theorem 1.9. Let $f=f_{d}+f_{d-1}+\cdots+f_{0}: \mathbb{C}^{n} \rightarrow \mathbb{C}$ be a polynomial function of degree $d$ with a finite number of critical points. Let $\mathcal{S}_{\infty}$ be a stratification as above then

$$
\mu(f)=(d-1)^{n}-\mu\left(\mathbb{P}_{\infty}^{n-1} ; D\right)-\sum_{S \in \mathcal{S}_{\infty}} \chi(S) \mu_{S}^{\infty}
$$

or

$$
\mu(f)+\lambda(f)=(d-1)^{n}-\mu\left(\mathbb{P}_{\infty}^{n-1} ; D\right)-\mu_{g e n}^{\infty}(f)
$$

Therefore if $\mu_{f}^{\infty}$ is constant then

$$
\mu(f)=(d-1)^{n}-\mu\left(\mathbb{P}_{\infty}^{n-1} ; D\right)-\mu_{f}^{\infty} .
$$

Proof. The main ingredient in this proof is (1.6). Let us take again $g:=$ $x_{1}^{d}+\cdots+x_{n}^{d}, \mu(g)=(d-1)^{n}$, and $D_{g}$ the smooth hypersurface of degree 
$d$ that is the intersection of the compactified fibres of $g$ with the hyperplane at infinity. Since $\mu_{g}^{\infty}$ has constant value equal to zero, see the proof of (1.7), one takes the trivial stratification of $\mathbb{C}$ for $g$. After (1.6) one has

$$
\mu(f)+\sum_{S \in \mathcal{S}_{\infty}} \chi(S) \mu_{S}^{\infty}+(-1)^{n-1} \chi(D)=(d-1)^{n}+(-1)^{n-1} \chi\left(D_{g}\right) .
$$

Using (1.1) we are done.

\section{Non-degeneracy and $\lambda(f)$}

For a generic complex polynomial function $f$ we will show that the Euler characteristic of the generic fibre of $f$ can be computed using the Newton number of the Newton polytope at infinity of $f$. Moreover this Newton number can be computed algorithmically.

As a consequence if $f$ has a finite number of critical points the invariant $\lambda(f)$ is obtained from the Newton number of the Newton polytope at infinity and the invariant $\mu(f)$. It is known how this invariant $\mu(f)$ is related to this polytope for convenient polynomials, see $[\mathrm{Ku}]$, and for two variable polynomials, see $[\mathrm{C}]$.

Let $f \in \mathbb{C}\left[x_{1}, \ldots, x_{n}\right]$ be a polynomial, $f=\sum a_{m} x^{m}, x^{m}=x_{1}^{m_{1}} \ldots$ $x_{n}^{m_{n}}$ with $f(0)=0$. Let $\operatorname{Supp}(f)=\left\{m \in \mathbb{N}^{n}: a_{m} \neq 0\right\}$ and let $\Gamma_{-}^{\infty}(f)$ be the convex closure of the set $\{0\} \cup \operatorname{Supp}(f)$ in $\mathbb{R}_{+}^{n}$. The Newton boundary at infinity of $f$, denoted by $\Gamma^{\infty}(f)$, is the polytope formed by the closed faces of $\Gamma_{-}^{\infty}(f)$ which do not contain the origin. The polynomial

$$
f^{\infty}=\sum_{m \in \Gamma^{\infty}(f)} a_{m} x^{m}
$$

is called the Newton principal part at infinity of $f$.

For each closed face $\gamma$ of the polytope $\Gamma^{\infty}(f)$ we denote by $f_{\gamma}$ the polynomial $\sum_{m \in \gamma} a_{m} x^{m}$. The Newton principal part at infinity of $f$ is nondegenerated on $\gamma$ if the system of equations

$$
\frac{\partial f_{\gamma}}{\partial x_{1}}=\cdots=\frac{\partial f_{\gamma}}{\partial x_{n}}=0
$$

has no solution in $\left(\mathbb{C}^{*}\right)^{n}$. When $f^{\infty}$ is non-degenerated on each face $\gamma$ of $\Gamma^{\infty}(f)$ we say that the Newton principal part at infinity of $f$ is nondegenerated.

Let $I \subset\{1, \ldots, n\}$. For any subset $A$ in $\mathbb{C}^{n}$ or $\mathbb{R}^{n}$ we denote by $(A)^{I}$ the set $\left\{\left(x_{1}, \ldots, x_{n}\right) \in A: x_{i} \neq 0\right.$ if only if $\left.i \in I\right\}$. For any polynomial $f \in$ $\mathbb{C}\left[x_{1}, \ldots, x_{n}\right]$ we denote by $f_{I}$ the polynomial $f$ where the indeterminate 
$x_{i}$ is zero whenever $i \notin I$. The proof of the next lemma is clear from the definitions.

Lemma 2.1. Let $f \in \mathbb{C}\left[x_{1}, \ldots, x_{n}\right]$ be a polynomial with non-degenerated Newton principal part at infinity. For every $I \subset\{1, \ldots, n\}$ then

(1) the polynomial $f_{I}$ has non-degenerated Newton principal part at infinity.

(2) $\quad \Gamma_{-}^{\infty}\left(f_{I}\right)=\Gamma_{-}^{\infty}(f) \cap \mathbb{R}_{+}^{I}$.

Let $S$ be a compact polytope in $\mathbb{R}_{+}^{n}$. The Newton number of $S$ is defined as follows

$$
\nu(S)=n ! V_{n}-(n-1) ! V_{n-1}+\ldots+(-1)^{n-1} V_{1}+(-1)^{n}
$$

where $V_{n}$ is the $n$-dimensional volume of $S$ and for all $q \in\{1, \ldots, n-1\}$, $V_{q}$ is the sum of the $q$-dimensional volumes of the intersection of $S$ with all $q$-dimensional coordinate planes.

Definition 2.2. The Newton number at infinity of $f$ is $\nu(f):=\nu\left(\Gamma_{-}^{\infty}(f)\right)$.

Let us recall another non-degeneracy condition associated with a polynomial. Let $h \in \mathbb{C}\left[x_{1}, \ldots, x_{n}\right]$ be a polynomial and let $\Delta(h)$ be the convex closure of $\operatorname{Supp}(h)$ in $\mathbb{R}_{+}^{n}$. The polynomial $h$ is called 0-non-degenerated, $[\mathrm{Ku}]$, or globally non-degenerated, [O], if for any face $\gamma$ of $\Delta(h)$ the system of equations

$$
h_{\gamma}=\frac{\partial h_{\gamma}}{\partial x_{1}}=\cdots=\frac{\partial h_{\gamma}}{\partial x_{n}}=0
$$

has no solution in $\left(\mathbb{C}^{*}\right)^{n}$.

Theorem 2.3. [BKK] [Kh] [O] Let $h \in \mathbb{C}\left[x_{1}, \ldots, x_{n}\right]$ be a 0-non-degene-rated polynomial then the Euler characteristic of the set $\left\{x \in\left(\mathbb{C}^{*}\right)^{n}\right.$ : $h(x)=0\}$ is equal to $(-1)^{n-1} n ! V_{n}(\Delta(h))$, where $V_{n}(\Delta(h))$ is the $n$ dimensional volume of $\Delta(h)$.

The proof of the following lemma is an easy consequence of the definitions.

Lemma 2.4. Let $h \in \mathbb{C}\left[x_{1}, \ldots, x_{n}\right]$ be a 0 -non-degenerated polynomial. Let $I \subset\{1, \ldots, n\}$, then

(1) the polynomial $h_{I}$ is 0-non-degenerated.

(2) $\Delta\left(h_{I}\right)=\Delta(h) \cap \mathbb{R}_{+}^{I}$.

Proposition 2.5. For any 0 -non-degenerated polynomial $h \in \mathbb{C}\left[x_{1}, \ldots, x_{n}\right]$ with $h(0) \neq 0$ following equality holds

$$
(-1)^{n-1}\left(\chi\left(\left\{x \in \mathbb{C}^{n}: h(x)=0\right\}\right)-1\right)=\nu(\Delta(h)) .
$$


Proof. Let $F \subset \mathbb{C}^{n}$ be the affine set

$$
F:=\left\{x \in \mathbb{C}^{n}: h(x)=0\right\} .
$$

For each $I \subset\{1, \ldots, n\}$, let us consider the affine set

$$
F_{I}^{*}:=F \cap\left(\mathbb{C}^{*}\right)^{I}=\left\{\left(x_{1}, \ldots, x_{n}\right) \in F: x_{i} \neq 0 \text { if only if } i \in I\right\} .
$$

So $F=\bigcup_{I \subset\{1, \ldots, n\}} F_{I}^{*}$ and $\chi(F)=\sum_{I \subset\{1, \ldots, n\}} \chi\left(F_{I}^{*}\right)$.

Let $V_{n}$ be the $n$-dimensional volume of $\Delta(h)$ and for $1 \leq q \leq n-1$, let $V_{q}$ be the $q$-dimensional volume of the intersections of $\Delta(h)$ with all $q$-dimensional coordinate planes. For every $q \in\{1, \ldots, n\}$ let us set $J_{q}:=$ $\{I \subset\{1, \ldots, n\}: \#(I)=q\}$. It is clear that

$$
\sum_{I \subset\{1, \ldots, n\}} \chi\left(F_{I}^{*}\right)=\sum_{q=1}^{n} \sum_{I \in J_{q}} \chi\left(F_{I}^{*}\right)
$$

For any $I \in J_{q}$ one has $F_{I}^{*}=\left\{x \in\left(\mathbb{C}^{*}\right)^{I}: h_{I}=0\right\}$. Since (2.1) $h_{I}$ has non-degenerated Newton principal part at infinity. Moreover lemma (2.4) shows that $h_{I}$ is a 0 -non-degenerated polynomial. Using (2.3) one gets the equality

$$
\chi\left(F_{I}^{*}\right)=(-1)^{q-1} q ! V_{q}\left(\Delta\left(h_{I}\right)\right)
$$

where $V_{q}\left(\Delta\left(h_{I}\right)\right)$ is the $q$-dimensional volume of $\Delta\left(h_{I}\right)$. For a given $q \in$ $\{1, \ldots, n\}$ one has

$$
\sum_{I \in J_{q}} \chi\left(F_{I}^{*}\right)=(-1)^{q-1} \sum_{I \in J_{q}} q ! V_{q}\left(\Delta\left(h_{I}\right)\right)=(-1)^{q-1} q ! V_{q} .
$$

This means that

$$
\chi(F)=\sum_{I \subset\{1, \ldots, n\}} \chi\left(F_{I}^{*}\right)=\sum_{q=1}^{n}(-1)^{q-1} q ! V_{q} .
$$

By adding -1 and multiplying by $(-1)^{n-1}$ on both sides of the above equality one gets

$$
(-1)^{n-1}(\chi(F)-1)=(-1)^{n-1} \sum_{q=0}^{n}(-1)^{q-1} q ! V_{q}=\nu(\Delta(h)) .
$$

Let $f: \mathbb{C}^{n} \rightarrow \mathbb{C}$ be a polynomial function. This map induces a locally trivial fibration $f: \mathbb{C}^{n} \backslash f^{-1}\left(B_{f}\right) \rightarrow \mathbb{C} \backslash B_{f}$ over the complement of the bifurcation set $B_{f}$. By Sard theorem for any $t \in B_{f}$ the fibre $F_{t}=f^{-1}(t)$ is a smooth manifold. In order to relate the Euler characteristic of a generic 
fiber $F_{t}$ with the Newton number at infinity of $f$ we need the following lemma which relates the above notions of non-degeneracy.

Lemma 2.6. If $f: \mathbb{C}^{n} \rightarrow \mathbb{C}$ is a complex polynomial function which has nondegenerated Newton principal part at infinity then there exists $t \in \mathbb{C} \backslash B_{f}$ such that $h:=f-t$ is a 0 -non-degenerated polynomial.

Proof. For every $t \in \mathbb{C} \backslash B_{f}$ it is clear that $\operatorname{Supp}(h)=\{0\} \cup S u p p(f)$. This means that the polytopes $\Delta(h)$ and $\Gamma_{-}^{\infty}(f)$ coincide. Moreover, for every closed face $\gamma$ of this polytope the equations

$$
\frac{\partial h_{\gamma}}{\partial x_{1}}=\cdots=\frac{\partial h_{\gamma}}{\partial x_{n}}=0
$$

are equivalent to

$$
\frac{\partial f_{\gamma}}{\partial x_{1}}=\cdots=\frac{\partial f_{\gamma}}{\partial x_{n}}=0 .
$$

Let $\gamma$ be a face of $\Delta(h)$.

(i) If $\gamma$ does not contain the origin then $h$ is 0 -non-degenerated on $\gamma$ because $f$ has non-degenerated Newton principal part at infinity.

(ii) If $\gamma$ contains the origin then applying Sard theorem there exists a dense open set $U_{\gamma}$ in $\mathbb{C}$ such that the polynomial map $f_{\gamma}: \mathbb{C}^{n} \cap f^{-1}\left(U_{\gamma}\right) \rightarrow U_{\gamma}$ has smooth fibres. Then for every $t \in U_{\gamma}$ the system of equations

$$
f_{\gamma}-t=\frac{\partial f_{\gamma}}{\partial x_{1}}=\cdots=\frac{\partial f_{\gamma}}{\partial x_{n}}=0
$$

has no solution.

Since the number of faces of $\Delta(h)$ is finite we have proved the lemma.

Theorem 2.7. Let $f: \mathbb{C}^{n} \rightarrow \mathbb{C}$ be a complex polynomial function. Let $t \in \mathbb{C} \backslash B_{f}$. If $f$ has non-degenerated Newton principal part at infinity then

$$
(-1)^{n-1}\left(\chi\left(F_{t}\right)-1\right)=\nu(f) .
$$

Proof. Let $f \in \mathbb{C}\left[x_{1}, \ldots, x_{n}\right]$ be a polynomial with non-degenerated Newton principal part at infinity. Let $t \in \mathbb{C} \backslash B_{f}$ and let $F_{t}=\left\{x \in \mathbb{C}^{n}: F(x)=t\right\}$ be a generic fibre, indeed a smooth fiber, such that $t$ verifies (2.6). Next we apply Proposition (2.5) and the result follows.

Corollary 2.8. Let $f: \mathbb{C}^{n} \rightarrow \mathbb{C}$ be a complex polynomial function with a finite number of critical points and with non-degenerated Newton principal part at infinity then

$$
\mu(f)+\lambda(f)=\nu(f) .
$$


Proof. It is a straightforward consequence of the theorems (2.7) and (1.7).

\section{Final remarks}

The Corollary (2.8) is a generalization of a Kushnirenko's result obtained under the additional hypothesis that $f$ is a convenient polynomial, see [Ku]. Moreover Pierrette Cassou-Noguès, [C], proved Corollary (2.8) for $n=2$. In both cases they obtained also the inequality $\leq$ whenever $f$ is degenerated at infinity. Since in the two variable polynomials case $\lambda(f)=0$ if $f$ is tame, $[\mathrm{H}]$, then she proved Kushnirenko's theorem for tame polynomials in two variables (non necessarily convenient) and she conjectured the same result in higher dimensions.

Conjecture.[C] Let $f: \mathbb{C}^{n} \rightarrow \mathbb{C}$ be a tame polynomial function then

(1) $\mu(f) \leq \nu(f)$.

(2) If $f$ has non-degenerated Newton principal part at infinity then

$$
\mu(f)=\nu(f) .
$$

Notice that Corollary (2.8) proves the equality part of the conjecture of Cassou-Noguès not only for tame polynomials but for any polynomial such that $\lambda(f)=0$. For instance this condition includes quasi-tame ([N1]) and M-tame ([NZ]) polynomials. Example 3.1 bellow proves that the inequality part of her conjecture is not true when $f$ is degenerated.

Finally we list some examples and open questions:

Example 3.1. Let $f=x+(x+y)^{2}$ and let $f: \mathbb{C}^{2} \rightarrow \mathbb{C}$ be the corresponding polynomial function. Since $f$ is a component of an automorphism of $\mathbb{C}^{2}$ then $f$ is a tame polynomial. Moreover $\nu(f)=1$ ( $f$ has degenerated principal part at infinity and the inequality holds). Now let $g$ be the same polynomial as $f$ but considered as a polynomial in three variables and let $g: \mathbb{C}^{3} \rightarrow \mathbb{C}$ be its corresponding function. As for $f$ the polynomial $g$ has $\mu(g)=\lambda(g)=0$ and it is tame, see [B, Prop 3.1]. Again $g$ has degenerated principal part at infinity and $\nu(g)=-\nu(f)=-1<\mu(g)+\lambda(g)$. Nevertheless (1) in Cassou-Noguès' conjecture is open for degenerated tame polynomials in exactly $n$ variables.

Example 3.2. Let us review the examples of Cassou-Noguès, [C] Sect. 6. Her first example $f=x(z-1)^{2} z+y^{4}+y z$ is valid to prove that the other inequality is possible. She proved that $\mu(f)=3, \lambda(f)=0$ and $\nu(f)=9$.

Example 3.3. Her second example $f=x y+x z^{3}+x^{2} z^{7}+y^{2} z^{3}+x y z^{6}$ has non-degenerated Newton principal part at infinity. Using SINGULAR, 
[GPS98], one gets $\mu(f)=20$. Moreover the Newton number at infinity of $f$ is $\nu(f)=23$ and then $\lambda(f)=3$ following Cassou-Noguès proof.

Example 3.4. Let $f=x+x^{2} y z$. The function $f: \mathbb{C}^{3} \rightarrow \mathbb{C}$ has no critical points, namely $\mu(f)=0$. Since $f$ has non-degenerated Newton principal part at infinity, the invariants $\lambda(f)$ and $\nu(f)$ are equal. Let us compute $\nu(f)=3 ! V_{3}-2 V_{2}+V_{1}-1$. The polytope $\Gamma_{-}^{\infty}(f)$ is the convex closure of the points $\{(0,0,0),(1,0,0),(2,1,1)\}$. So it is a two-dimensional polytope and then $V_{3}=0$. Moreover any intersection of $\Gamma_{-}^{\infty}(f)$ with any coordinate plane has not dimension two, i.e. $V_{2}=0$. Finally $V_{1}=1$ and $\lambda(f)=\nu(f)=0$. But it is clear that the fibre $F_{0}$ is not topologically equivalent to any other fibre. This example shows that $\lambda(f)=0$ does not imply $B_{f}=\emptyset$.

Example 3.5. Let $f=x+x^{2} y^{2}+x^{3} z$. Again in this case $\mu(f)=0$ and it has non-degenerated Newton principal part at infinity. Then $\lambda(f)=\nu(f)=-1$. In this case $V_{3}=\frac{1}{3}, V_{2}=\frac{1}{2}+1$ and $V_{1}=1$. This example shows how the striking relationship between $\mu(f)$ and $\nu(f)$ is corrected by the integer $\lambda(f)$. A family of this sort of examples is given by $f_{a}=x+x^{2} y^{a}+x^{a+1} z$. Moreover with $f$, or $f_{a}$, and the Sebastiani-Thom-type formula for $\lambda(f)$ one can get polynomials with $\mu(f)=0$ and $\lambda(f)$ as negative as desired. Nevertheless we have the following

- Conjecture. There does not exist a polynomial $f$ such that $\mu(f)>0$ and $\lambda(f)<0$.

Example 3.6. Next example shows that the hypothesis " $f$ has isolated critical points" is necessary. Let $f=x+x^{2} y z+y z$. This polynomial has nondegenerated Newton principal part at infinity, it is not convenient, of course $\nu(f)$ is finite but the set of critical values is not finite, i.e., $\mu(f)$ does not exist.

Example 3.7. We study a family of polynomials $f=x^{a} y^{b}\left(x^{c} y^{d}-z^{c+d}\right)+z$. When $b c-a d \neq 0$ the polynomial $f$ has no critical points, i.e., $\mu(f)=0$ and in this case $\nu(f)=|b c-a d|$. So after corollary (2.8) $\lambda(f)=|b c-a d|$.

Example 3.8. Finally let us take $g_{k}=x+x^{2} y z+v^{k}$. The Sebastiani-Thomtype formulæ for $\mu$ and $\lambda$ show that $\mu\left(g_{k}\right)=\lambda\left(g_{k}\right)=0$; then we have examples of all fibre-irreducible-polynomials where the Euler characteristic of the fibres is constant. From [N2] one knows that the generic fibre $G_{k}$ of $g_{k}$ has the homotopy type of the join of the generic fibres of $x+x^{2} y z$ and $v^{k}$. Applying the formula of the homology of a join space, see [D, p. 88], one has

$$
H_{i}\left(G_{k}, \mathbb{Z}\right) \cong \begin{cases}\mathbb{Z} & \text { if } i=0, \\ \mathbb{Z}^{k-1} & \text { if } i=2,3 \\ 0 & \text { otherwise }\end{cases}
$$


Moreover the generic fibre $G_{k}$ is simply connected. These facts prove that $G_{k}$ is neither contractible nor a wedge of 3-spheres; in particular it has not $\mathcal{W}$-isolated singularities at infinity in the sense of [ST]. Related to the condition $\mu(f)=\lambda(f)=0$ one has the conjecture of Siersma-Tibăr:

Conjecture [ST]. Let $f: \mathbb{C}^{n} \rightarrow \mathbb{C}$ be a polynomial function with $\mathcal{W}$ isolated singularities at infinity and $\mu(f)=\lambda(f)=0$, then $f$ is equivalent to a linear function module an isomorphism of $\mathbb{C}^{n}$.

The polynomial $g_{k}$ shows that one can not drop the condition $\mathcal{W}$-isolated singularities at infinity in the above conjecture. The value $0 \in \mathbb{C}$ is the only atypical value of the polynomial function $g_{k}$. Let $G_{k}^{0}$ be the special fibre over 0 . Its fundamental group is cyclic of order $k$. The computations on the fundamental group may be performed by means of the restriction of the projection on $x, y, z$. The computation of its homology groups gives:

$$
H_{i}\left(G_{k}^{0}, \mathbb{Z}\right) \cong \begin{cases}\mathbb{Z} & \text { if } i=0, \\ \mathbb{Z} / k \mathbb{Z} & \text { if } i=1,2 \\ 0 & \text { otherwise. }\end{cases}
$$

Applying a result of Kaliman, see [K, Theorem A] or [Z, Theorem 6.9], one has that if $k, l$ are coprime positive numbers then the polynomial

$$
h_{k, l}(x, y, z, u, v):=x+x^{2} y z+u^{k}+v^{l}
$$

verifies also that Euler characteristic of the fibres is constant and in this case the (special) fibre over 0 , denoted by $H_{k, l}$, is contractible and hence diffeomorphic to $\mathbb{C}^{4}$. These polynomials are not topologically trivial (the generic fibre is not contractible) despite of the fact that the Euler characteristic of the fibres is a constant function.

The 4 -folds $H_{k, l}$ are related to the 3 -folds

$$
F_{k, l}:=\left\{(x, y, u, v) \in \mathbb{C}^{4} \mid x+x^{2} y+u^{k}+v^{l}=0\right\}
$$

introduced by M. Koras and P. Russell in [KR] and studied by MakarLimanov and Kaliman; it is known, see $[\mathrm{M}]$ and $[\mathrm{KM}]$, that $F_{k, l}$ is an exotic $\mathbb{C}^{3}$.

- Conjecture. $H_{k, l}$ is an exotic $\mathbb{C}^{4}$.

A negative answer would give a counterexample to the AbhyankarShataye conjecture in dimensions 4 and 5 . By blowing-up a closed subvariety of $V:=H_{k, l} \cap\{x=0\}$, as in [KR, Prop. 7.8], one can get a morphism $\pi: \mathbb{C}^{4} \rightarrow H_{k, l}$ which is an isomorphism on $H_{k, l} \backslash V$, in particular $\pi$ is a dominant map. Note also that the map

$$
\alpha_{k, l} H_{k, l} \rightarrow \mathbb{C}: \quad \alpha_{k, l}(x, y, z, u, v)=z
$$


verifies that its generic fibre is isomorphic to $F_{k, l}$ and the fiber over 0 is $\mathbb{C}^{3}$. We also note that the symmetry of $y, z$ induces a 2 -fold ramified covering of $H_{k, l}$ onto $F_{k, l} \times \mathbb{C}$; it is not known, see [KM], whether $F_{k, l} \times \mathbb{C}$ has an exotic structure.

\section{References}

[ALM] E. Artal Bartolo, I. Luengo, A. Melle-Hernández, On the topology of a generic fibre of a polynomial map, Comm. in Algebra (to appear)

[BKK] D. Bernstein, A.G. Khovanskii, A.G. Kushnirenko, Newton polyhedra, Usp. Mat. Nauk 31 (1976), 201-202

[B] S.A. Broughton, Milnor numbers and the topology of polynomial hypersurfaces, Invent. math. 92 (1988), 217-241

[C] P. Cassou-Noguès, Sur le généralisation d'un théorème de Kushnirenko, Compositio Math. 103 (1996), 95-121

[CD] P. Cassou-Noguès, A. Dimca, Topology of complex polynomials via polar curves, Prépublications de l'Université de Bordeaux I 82 (1998),

[D] A. Dimca, Singularities and topology of hypersurfaces, Universitext, SpringerVerlag, New York, 1992

[Du] A.H. Durfee, Five definitions of Critical Point at Infinity, Singularities (Oberwolfach, 1996), Progr. Math., 162, Birkhäuser, Basel, 1998, pp. 345-360

[GPS98] G.M. Greuel, G. Pfister, H. Schöenemann, Singular, version 1.2 User Manual, Reports On Computer Algebra, Centre for Computer Algebra, University of Kaiserslauten, http://www.mathematik.uni-kl.de/zzca/Singular, 1998

[GLM1] S.M. Gusein-Zade, I. Luengo, A. Melle-Hernández, On zeta-function of a polynomial at infinity, Bull. Sci. Math. (to appear)

[GLM2] S.M. Gusein-Zade, I. Luengo, A. Melle-Hernández, On atypical values and local monodromies of meromorphic functions, Proceedings of the Stetklov Mathematical Institute (to appear)

[H] H.V. Hà, Nombres de Lojasiewicz et singularitès à l'infini des polynômes de deux variables complexes, C.R. Acad Sci. Paris 311 (Série I) (1990), 429-432

[HL] H.V. Hà, D.T. Lê, Sur la topologie des polynômes complexes, Acta Math. Vietnamica 9 (1984), 21-32

[K] S. Kaliman, Smooth contractible hypersurfaces in $\mathbb{C}^{n}$ and exotic algebraic structures on $\mathbb{C}^{3}$, Math. Z. 214 (1993), 499-510

[KM] S. Kaliman, L. Makar-Limanov, On the Russell-Koras contractible threefolds, J. Algebraic Geometry 6 (1997), 247-268

[Kh] A.G. Khovanskii, Newton polyhedra and genus of complete intersections, Funct. Anal. Appl. 12 (1978), 38-46

[KR] M. Koras, P. Russell, Contractible threefolds and $\mathbb{C}^{*}$-actions on $\mathbb{C}^{3}$, J. Algebraic Geometry 6 (1997), 671-695

[Ku] A.G. Kushnirenko, Polyèdres de Newton et nombres de Milnor, Invent. math. 32 (1976), 1-31

[M] L. Makar-Limanov, On the hypersurface $x+x^{2} y+z^{2}+t^{3}=0$ in $\mathbb{C}^{4}$ or a $\mathbb{C}^{3}$-like threefold which is not $\mathbb{C}^{3}$, Israel J. Math. 96 (1996), 419-429

[Me] A. Melle-Hernández, Milnor numbers of surfaces, Israel J. Math. (to appear)

[N1] A. Némethi, Théorie de Lefschetz pour les variétés algébriques affines, C.R. Acad. Sci. Paris Sér. I Math. 303 (1986), 567-570 
[N2] A. Némethi, Global Sebastiani-Thom theorem for polynomial maps, J. Math. Soc. Japan 43 (1991), 213-218

[NZ] A. Némethi, A. Zaharia, On the bifurcation set of a polynomial function and Newton boundary, Publ. RIMS Kyoto Univ. 26 (1990), 681-689

[O] M. Oka, On the topology of the Newton boundary II (generic weighted homogeneous singularity), J Math. Soc. Japan 32 (1980), 65-92

[P1] A. Parusiński, A generalization of the Milnor number, Math. Ann. 281 (1988), 247-254

[P2] A. Parusiński, Multiplicity of the dual variety, Bull. London Math. Soc. 23 (1991), 428-436

[P3] A. Parusiński, On the bifurcation set of a complex polynomial with isolated singularities at the infinity, Compositio Math. 97 (1995), 369-384

[PP] A. Parusiński, P. Pragacz, A Formula for the Euler characteristic of singular hypersurfaces, J. Algebraic Geometry 4 (1995), 337-351

[ST] D. Siersma, M. Tibăr, Singularities at infinity and their vanishing cycles, Duke Math. J. 80 (1995), 771-783

[T] M. Tibăr, Regularity at infinity of real and complex polynomials functions, Singularity Theory, C.T.C. Wall Anniversary Volume, Cambrige Univ. Press, Cambridge, (to appear)

[V] A.N. Varchenko, Theorems on the topological equisingularity of families of algebraic varieties and families of polynomials mappings, Math. USSR Izvestija 6 (1972), 949-1008

[Ve] J.L. Verdier, Stratifications de: Whitney et théorème de Bertini-Sard, Inv. math. 36 (1976), 295-312

[Z] M. Zaidenberg, On exotic algebraic structures on affine spaces, Geometric Complex Analysis, J. Noguchi et a. eds., World Scientific Publ. Co., Singapore, 1996, pp. 691-714 